Polymer Journal, Vol. 39, No. 10, pp. 1025-1029 (2007)

(C) 2007 The Society of Polymer Science, Japan

\title{
Nanocables Prepared from Polyamide 66 nanotubes Enveloping Pt nanowires by a Secondary-template Method
}

\author{
X. L. She, ${ }^{1}$ G. J. SONG,${ }^{1, \dagger}$ Z. Peng, ${ }^{1}$ J. J. LI, ${ }^{1}$ C. T. LIM, ${ }^{2}$ \\ E. P. S. TAN, ${ }^{2}$ L. $\mathrm{LV}^{3}{ }^{3}$ and X. S. ZHAO ${ }^{3 \dagger}$ \\ ${ }^{1}$ Institute of Polymer Materials, Qingdao University, Qingdao, P. R. China 266071 \\ ${ }^{2}$ Department of Mechanical Engineering \& Division of Bioengineering, \\ National University of Singapore, Singapore 119260 \\ ${ }^{3}$ Department of Chemical and Biomolecular Engineering, National University of Singapore, Singapore 119260
}

(Received April 14, 2007; Accepted June 27, 2007; Published August 7, 2007)

\begin{abstract}
Polyamide 66 (PA66) nanotubes were prepared by filtrating a PA66 solution into pores of anodic aluminum oxide (AAO) template. The AAO/PA66 nanotubes composite membrane can be used as a secondary template. Pt nanowires were then fabricated in the polymer nanotubes of the secondary template by electrochemical deposition method. Nanocables from Polyamide 66 nanotubes embedded Pt nanowires were successfully obtained. Scanning electron microscopy (SEM) and Transmission electron microscope (TEM) were employed to characterize the structure and morphology of one-dimension nanomaterials. We find that the polymer nanotubes enveloping Pt nanowires have a higher thermal-decomposition temperature than the bulk counterpart. The unique property will be beneficial to their application under a higher temperature. [doi:10.1295/polymj.PJ2007008]

KEY WORDS Nanocables / Nanotubes / AAO / Secondary-template Method /
\end{abstract}

One-dimensional (1D) nanostructure has been a subject of intensive research interest because of its unique properties and potential applications. ${ }^{1}$ In particular, nanowires, ${ }^{2,3}$ nanotubes, ${ }^{4-6}$ and nanocables ${ }^{7,8}$ have attracted considerable attention. Martin and his co-workers $^{9-12}$ were the first who used AAO membrane-based-on-template method to prepare 1D nanomaterials. Subsequent studies have reported that various nanomaterials such as nanotubes and nanowires composed of metals, semiconductors, polymers, and their composites can be fabricated using the template synthesis method. ${ }^{13}$ These $1 \mathrm{D}$ nanostructures can be used as nanodevices for nanotechnology. ${ }^{14}$

However, metal nanowires are sensitive to many environments such as high temperature, air and humidity. Even the noble metal platinum nanowires react easily owing to their nanostructure, which seriously limits their application in many fields. So a polymer sheath can be used to enclose the metal nanowires to protect them from oxidation and corrosion. Previously, conducting polymers have been used to protect metal nanowires. ${ }^{13,15-18}$ The polymers are seriously reactive with oxygen due to the conjugated structure. Although some non-conducting polymers have been reported using to protect the metal nanowires, the results are not perfect because of the intricate preparing process and the prepolymer they used. For example, Poly ( $p$-xylylene) nanotubes were used to protect the Pd nanowires. ${ }^{19-21}$

Recently, we have prepared several polymer nano- tubes into AAO template nanopores via a simple solution wetting technique. ${ }^{22-24}$ The obtained PA66 nanotubes enveloped into AAO can be used as a "secondary template", into which the metal nanowires are prepared by electrochemical deposition. The polymer/metal nanocables are formed at last. This technique for preparing nanocables can be defined as secondary template method. Many nanocables have been fabricated in our lab in this way. Herein, taking PA66/ Pt nanocables for example, the secondary template method was clearly demonstrated. It is believed that the method described here can be widely used in preparing other polymer/metal nanocables. The special structure of PA66/Pt nanocables was characterized by FESEM and TEM. In addition, their superior thermal stability and anti-solvent property were occasionally found, which endowing the nanocables many promising applications in nanodevices.

\section{EXPERIMENTAL PARTS}

\section{Preparation of Polymer Nanotubes}

Porous AAO template (purchased from Whatman International Ltd.) with an average pore diameter of $250 \mathrm{~nm}$ was treated in $\mathrm{CH}_{2} \mathrm{Cl}_{2}$ in an ultrasonic bath to clean its surface before used. A PA66 (from BASF) polymer solution (5.0 wt. \%) was prepared by dissolving PA66 pallets in formic acid $(\mathrm{HCOOH})$. One drop of the polymer solution was applied on a glass slide, and then the AAO membrane was placed on the solu-

${ }^{\dagger}$ To whom correspondence should be addressed (E-mail: xlshe@126.com). 


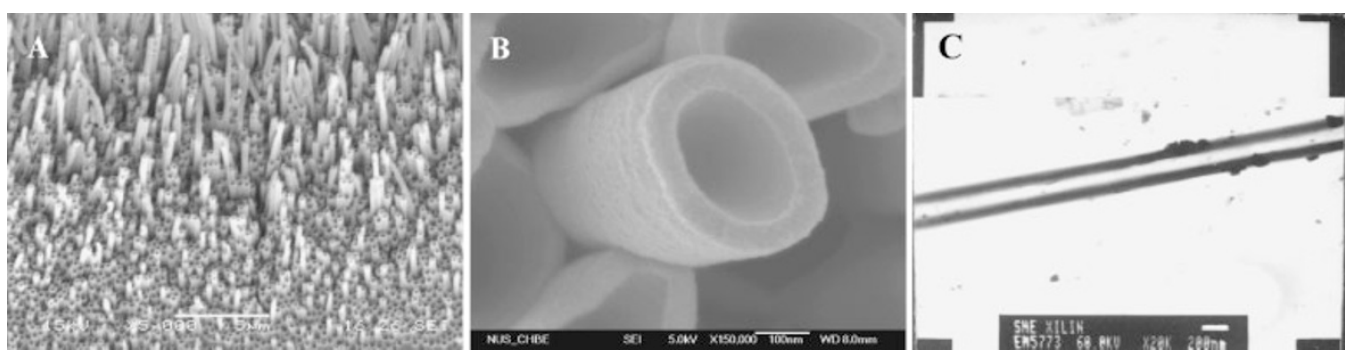

Figure 1. SEM and TEM images of the PA66 nanotube array (A) and a single nanotube (B and C).

tion drop. And the wetting process occurred on the pore surface of the AAO template. After $1 \mathrm{~min}$, the solvent evaporated completely to leave PA66 nanotubes on the surface of the template pores. The PA66/AAO composite membrane was removed from the slide by soaking it in hot water and then was put the membrane into $3 \mathrm{M}$ sodium hydroxide solution to remove the AAO template. Thus the polymer nanotubes and their array were successfully prepared. ${ }^{25}$

\section{Preparation of Polymer/metal Nanocables}

After removing the polymer on the surface of the template, the PA66/AAO composite membrane can be used as a "secondary template". It was fixed on a piece of conducting glass using $\mathrm{Ag}$ conducting glue to prepare a working electrode of tri-electrode system for electrochemical deposition. Pt nanowires were deposited into the PA66 nanotubes with a solution containing $0.2 \mathrm{mM} \mathrm{H}_{4} \mathrm{PtCl}_{6}, 0.2 \mathrm{M} \mathrm{NaH}_{2} \mathrm{PO}_{4}$, and $0.2 \mathrm{M}$ $\mathrm{Na}_{2} \mathrm{HPO}_{4}$ in the tri-electrode system. Electrochemical deposition was carried out at a constant voltage of $-0.5 \mathrm{~V}$ using a $\mathrm{CH}-1$ Potentiostat with a piece of $\mathrm{Pt}$ as the anode and a Calomel Electrode as the contrastive electrode. After the removal of the AAO template in a $3 \mathrm{M}$ sodium hydroxide solution, the nancables and their array were also obtained.

\section{Characterization of Nanomaterials}

Scanning Electron Microscopy (SEM). The AAO membrane with 1D nanomaterials was fixed on a microscope slide and placed in the $3 \mathrm{M}$ sodium hydroxide solution to dissolve the alumina partly or completely. The surface of the sample was then sputtered with $\mathrm{Au}$ and imaged with a JEOL JSM-840 or FESEM-6700 scanning electron microscope.

Transmission Electron Microscope (TEM). The AAO membrane with 1D nanomaterials was placed in the $3 \mathrm{M}$ sodium hydroxide solution for $48 \mathrm{~h}$ to remove the template entirely, and then stirred by supersonic wave to separate nanometer array into single nanotube, nanowire and nanocable. This solution was dropped on a microscope copper grid coated with holey carbon support film and imaged with a JEOL
JEM-2000EXII transmission electron microscope.

Thermal Stability and Anti-solvent Property. The nanocables with no template were placed into formic acid $(\mathrm{HCOOH})$ or pyrolysed in a muffle at different temperatures to remove their polymer sheath. The residual Pt wires were collected on a rounded AAO membrane by filtering and characterized by SEM.

\section{RESULTS AND DISCUSSION}

The SEM and TEM images of PA66 nanotubes are shown in Figure 1. Figure 1A is the PA66 nanotubes prepared from PA66 solution and their array looks like a brush. From the picture, the mouth and the $300 \mathrm{~nm}$ outer diameter of the PA66 nanotubes can be observed clearly. In addition, we can see that the nanotubes in the upside of Figure 1A are not regular and uniform. The reasons are as follows: First, after removing AAO template from the composite membrane, the carrier of nanotubes (double glue) could be rucked up in the process of preparing samples, and the nanotube array could be destroyed. Second, during the preparation of polymer nanotubes, we usually drop a little solution (about $1 \mu \mathrm{L}$ ) on the microscope glass in order to obtain entirely hollow nanotubes and to observe them clearly. $1 \mu \mathrm{L}$ solution only wets template partially, so there must be a transition area of polymer nanotubes on the AAO template. Figure $1 \mathrm{~A}$ only shows the transition area where the height of the nanotubes is different.

Figure 1B shows a single PA66 nanotube. From it, the PA66 nanotube and its $300 \mathrm{~nm}$ outer diameter can be observed clearly. The outer diameter is a bit larger than that of the AAO template pores for following reasons. Firstly, according to Ai's research results, polymer nanotubes may be swollen in $\mathrm{NaOH}$ solution during the process of removing AAO template. ${ }^{26} \mathrm{Sec}-$ ondly, there is some stress in the polymer nanotubes because polymer nanotubes are formed in a limited space of template nanopores. When the rigid wall of template was removed, the nanotubes will swell to a certain extent to release the stress. Thirdly, the templates are sold as having pores of $200 \mathrm{~nm}$, but measurement shows that the inner pore diameter is more 

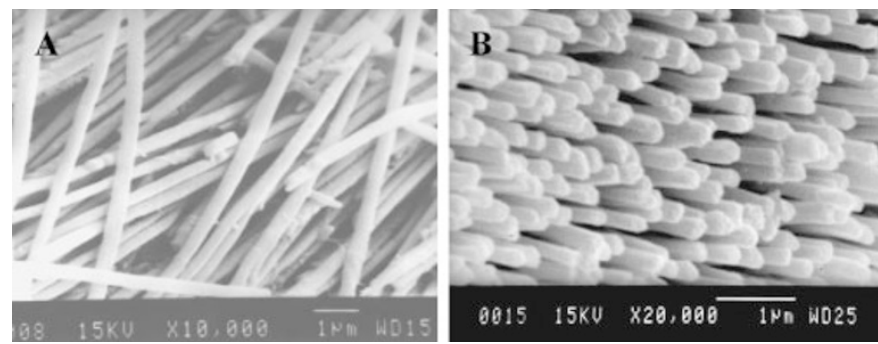

Figure 2. SEM images of the Pt nanowires prepared directly into pores of AAO template. (A) Pt nanowires deposited into a blank AAO template. (B) The array of Pt nanowires after removing partial AAO template.

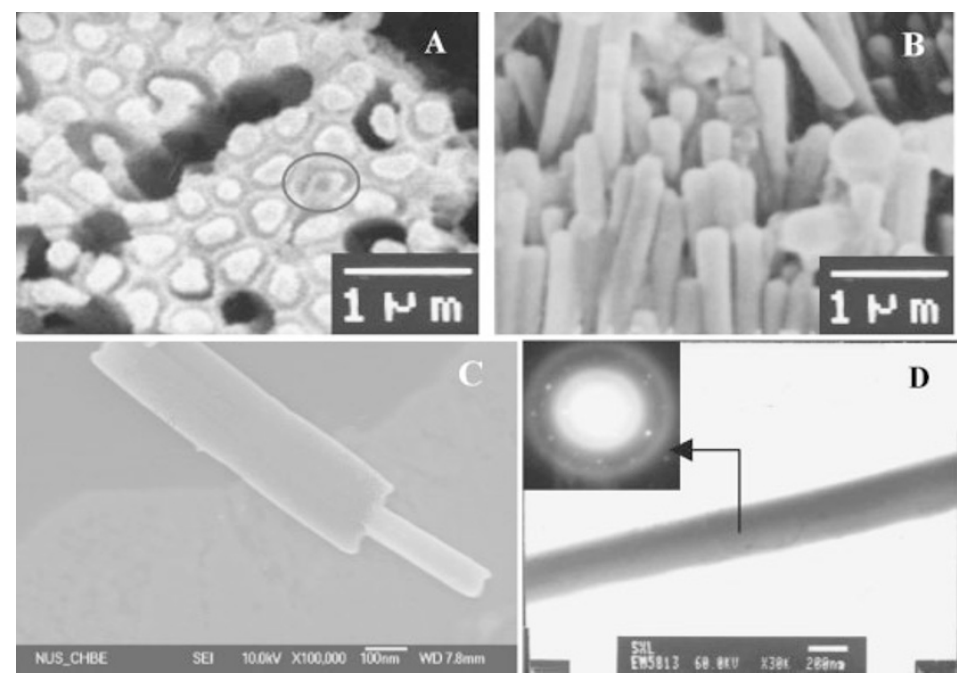

Figure 3. SEM and TEM images of the PA66/Pt nanocables and their array. (A) The top-view of PA66/Pt nanocables embedded into template nanopores. (B) The side-view of PA66/Pt nanocables. (C) A single nanocable removed partially polymer sheath (D) TEM of a single nanocable.

than $250 \mathrm{~nm}$ and their two surfaces have different porous diameters.

The TEM image of a single PA66 nanotube shown in Figure $1 \mathrm{C}$ reveals that the tube diameter is similar to that of the nanotubes obtained from the SEM image. It also shows that the surface of the PA66 nanotube contains some lumps, which are due to the residual alumina salt formed during the removal of the AAO template. Nevertheless, the TEM image clearly confirms that the nanotube is entirely hollow and the interior diameter is uniform throughout the whole tube, which is in good agreement with the Li's results. $^{27}$

Figure 2 shows the morphology of Pt nanowires and their array. From these two images, we can see that the nanowires have the external diameter of $c a$. $300 \mathrm{~nm}$, indicating that they were replicated from the template. A few of nanowires have a diameter more than $300 \mathrm{~nm}$ because the template's pore diameter is more than $300 \mathrm{~nm}$ and its two surfaces have different porous diameter. In Figure 2A, some smooth Pt nanowires are unyielding, which confirms they have good mechanical property. After removing partial template, a uniform and big-area nanowire array is obtained (see in Figure 2B).

Figure 3A presents the top-view of the PA66/Pt nanocables embedded into the nanopores of AAO template. It can be seen that all polymer nanotubes have been fully filled with $\mathrm{Pt}$ nanowires except for one nanotube in the red circlet. This may be due to pore blocking of a polymer nanotube mouth, through which the Pt precursor entered into polymer nanotube during the electrochemical deposition process. After removing the AAO template, the PA66 enveloping Pt nanocables were entirely disclosed in Figure 3B. Comparing to the polymer nanotubes, these cables became plump and unyielding like Pt nanowires (see in Figures $2 \mathrm{~A}$ and $3 \mathrm{~B}$ ).

Figure $3 \mathrm{C}$ clearly reveals the real "shell and core" structure of PA66/Pt nanocables. When preparing the nanocables into pores of AAO template, partial template was first removed by $\mathrm{NaOH}$ solution from upside to downside, then the counterpart of nanocables was disclosed partially. The sample was placed into formic acid to dissolve the disclosed polymer sheath. When the residual alumina template was also removed, the 


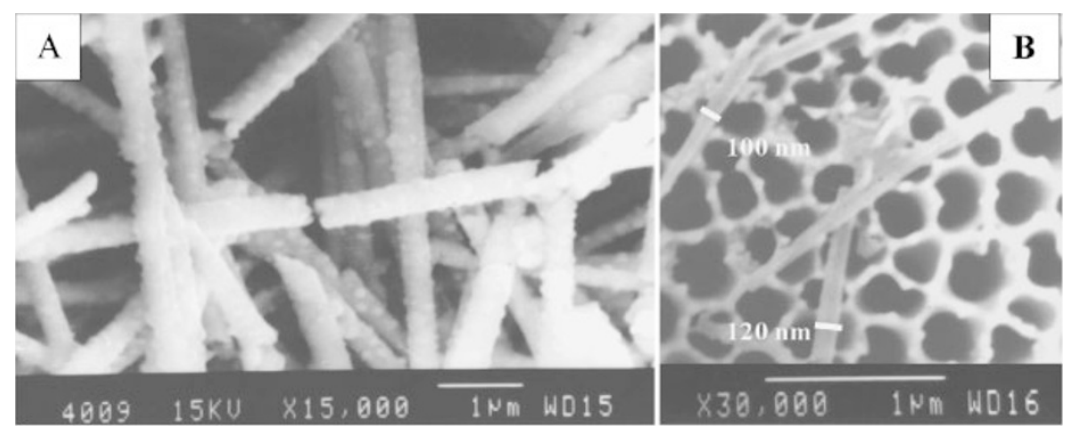

Figure 4. (A) PA66/Pt nanocables soaked into formic acid (HCOOH) for $2 \mathrm{~h}$. (B) The Pt nanowires after removing polymer sheath from the PA66/Pt nanocables in formic acid $(\mathrm{HCOOH})$ for $72 \mathrm{~h}$.
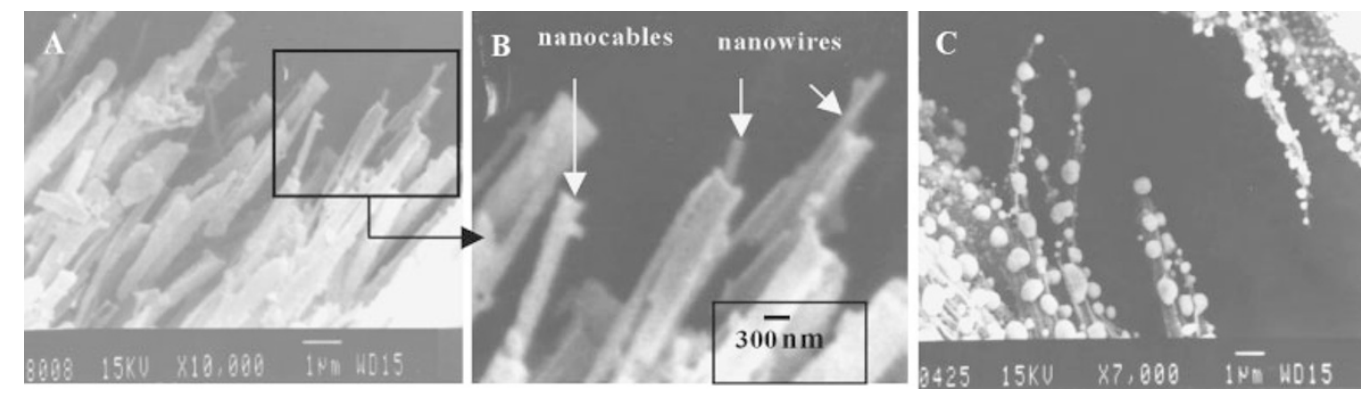

Figure 5. (A) SEM images of PA66/Pt nanocables after soaking in formic acid. (B) The magnification of part region of (A). (C) SEM images of PA66/Pt nanocables calcined at 600 Celsius.

clear "shell enveloped core" structure of nanocable was obtained finally (see in Figure 3C). From it, we can see that the cable was enclosed by the polymer nanotubes with more than $200 \mathrm{~nm}$ outer diameter and the diameter of Pt nanowire was about $100 \mathrm{~nm}$.

Figure 3D shows that the inner white channel of polymer nanotubes has disappeared, which fully verifies the nanotubes were filled with Pt. Another proof of the Pt presence is provided by the electron diffraction pattern collected by a nanobeam diffraction (NBD) mode with a beam converged to a $2 \mathrm{~nm}$ probe diameter (insert image in Figure 3D). The bright circle reveals of the polymer's polycrystalline while the bright dots represent the mono-crystalline structure of Pt metal.

Figure 4A shows the SEM image of the PA66/Pt nanocables after soaking into $\mathrm{HCOOH}$ for $2 \mathrm{~h}$. It can be observed that the surface of nanocables merely became coarse owing to the slight swelling of the polymer sheath in formic acid. It means that the polymer sheath have superior anti-solvent property compared with bulk PA66 which can easily dissolve in formic acid in $10 \mathrm{~min}$. The unique property may be arised from the special crystalline structure of the wall of polymer nanotubes. When the soaking time was prolonged to $72 \mathrm{~h}$, the polymer sheath was completely dissolved away, leaving behind $\mathrm{Pt}$ nanowires with the diameter of 100 120 nm (see in Figure 4B). Obviously, the diameter of the $\mathrm{Pt}$ nanowires is similar to the inner diameter of the PA66 nanotubes, indicating that $\mathrm{Pt}$ nanowires are just the cores of nanocables and the polymer nanotubes are their shells. The result also confirms the "shell and core" structure of these nanocables.

The Pt core-PA66 shell nanocable structure was further characterized. Figure $5 \mathrm{~A}$ and $\mathrm{B}$ show the SEM images of the PA66/Pt nanocables after soaking $30 \mathrm{~min}$ in formic acid. From Figure 3B, it can be clearly seen that the cables have a diameter of about $250 \mathrm{~nm}$ while the $\mathrm{Pt}$ wires in the cables exhibit a diameter of about $120 \mathrm{~nm}$. After calcination of the nanocables at 600 Celsius for $30 \mathrm{~min}$ in a muffle furnace, the polymer sheath became nano-spherical clusters along every strand of the nanowires (see in Figure 5C). Such interesting phenomenon is reported for the first time. Currently, we are investigating the properties of the globules. According to Li et al. ${ }^{27}$ polymer nanotubes display a higher thermal-decomposition temperature than the bulk counterpart. So the polymer/Pt nanocables may be applied under a higher temperature.

According to the discussion above, polymer nanotubes can be used for protecting metal nanowires to prepare a kind of nanocable-polymer nanotubes embedded metal nanowires. The polymer/metal nanocables will be applied in nanodevice preparation for their "polymer sheath and metal core" structure. In addition, the special microstructure of the wall of 
polymer nanotubes are endued with many superior properties such as thermal stability and anti-solvent property, which will widen polymer/metal nanocables' applications. ${ }^{28}$

\section{CONCLUSION}

In summary, we have demonstrated the "secondary template" approach to prepare polymer/metal nanocables using PA66/Pt system as an example. The method described here is applicable to the preparation of normal polymer nanotubes into the commercial AAO with pores of $250-\mathrm{nm}$. So it overcomes many limitations of previously established preparation methods, such as requiring AAO with larger pores (not less than $400 \mathrm{~nm}$ ) and special polymer. Using the polymer nanotubes embedded in AAO template as a "secondary template", we have fabricated Pt nanowires in the PA66 nanotubes via electrochemical deposition method. The PA66/Pt nanocables offer the possibility of using normal polymer nanotubes in nanodevices and provide an effective method for protecting metal nanowires from oxidation and corrosion. Such composite nanometer structure will be promising in the field of microelectronic devices and may be used in biosensors, biocatalysis, biorecognition, and drug delivery.

Acknowledgment. The authors thank the National Nature Science Foundation of China (No 50473012) for support and thank Dr Lu Lv, Zuocheng Zhou, Fabing Su, Wanping Guo, Xiaoying Bao and Yan Qingfeng for a valuable discussion and hearty help during I worked in National University of Singapore (NUS).

\section{REFERENCES}

1. Y. L. Wang, X. C. Jiang, and Y. Xia, J. Am. Chem. Soc., 125, 16176 (2003).

2. S. I. Moon and T. J. McCarthy, Macromolecules, 36, 4253 (2003).

3. F. Qian, Y. Li, D. L. Wang, C. J. Barrelet, and C. M. Lieber, Nano Lett., 4, 1975 (2004).
4. S. H. Joo, S. J. Choi, I. Oh, J. Kwak, Z. Liu, O. Terasaki, and R. Ryoo, Nature, 412, 169 (2001).

5. M. Steinhart, S. Senz, and R. B. Wehrspohn, Macromolecules, 36, 3646 (2003).

6. Y. Z. Long and L. J. Zhang, Macromol. Rapid Commun., 24, 938 (2003).

7. Y. Zhang, K. Suenaga, C. Colliex, and S. Lijiama, Science, 281, 973 (1998).

8. G. W. Ho, A. S. Wong, A. T. Wee, and M. E. Welland, Nano Lett., 4, 2023 (2004).

9. C. R. Martin, Science, 266, 1961 (1994).

10. G. Che, B. B. Lakshmi, C. R. Martin, E. R. Fisher, and R. S. Ruoff, Chem. Mater., 10, 260 (1998).

11. V. M. Cepak and C. R. Martin, Chem. Mater., 11, 1363 (1999).

12. S. F. Hou, C. C. Harrell, L. Trofin, P. Kohli, and C. R. Martin, J. Am. Chem. Soc., 126, 5674 (2004).

13. H. Q. Cao, Z. Xu, and D. Sheng, Adv. Mater., 13, 121 (2001).

14. S. Stewart and G. J. Liu, Angew. Chem., Int. Ed., 39, 340 (2000).

15. H. Q. Cao, Z. Xu, D. Sheng, J. M. Hong, H. Sang, and Y. W. Du, J. Mater. Chem., 11, 958 (2001).

16. M. Bognitzki, H. Q. Hou, M. Ishaque, T. Frese, M. Hellwig, C. Schwarte, A. Schaper, J. H. Wendorff, and A. Greiner, Adv. Mater., 12, 637 (2000).

17. M. Steinhart, J. H. Wendorff, and A. Greiner, Science, 296, 1997 (2002).

18. H. Q. Cao, C. Y. Tie, and Z. Xu, Appl. Phys. Lett., 78, 1592 (2001).

19. H. Q. Hou, Z. Jun, A. Reuning, A. Schaper, J. H. Wendorff, and A. Greiner, Macromolecules, 35, 2429 (2002).

20. Z. Li and G. J. Liu, Langmuir, 19, 10480 (2003).

21. J. S. Yu, J. Y. Kim, S. Lee, J. K. Mbindyo, B. R. Martin, and T. E. Mallouk, Chem. Commun., 24, 2445 (2000).

22. G. J. Song, X. L. She, Z. F. Fu, and J. J. Li, J. Mater. Res., 19, 3324 (2004).

23. J. J. Li, G. J. Song, and X. L. She, Polym. J., 38, 554 (2006).

24. X. L. She, G. J. Song, J. J. Li, P. Han, S. J. Yang, S. L. Wang, and Z. Peng, Polym. J., 38, 639 (2006).

25. X. L. She, G. J. Song, J. J. Li, P. Han, S. J. Yang, and Z. Peng, J. Mater. Res., 21, 1209 (2006).

26. S. F. Ai, G. Lu, Q. He, and J. B. Li, J. Am. Chem. Soc., 125, 11140 (2003).

27. B. S. Li and G. S. Zhu, Polym. Prepr., 43, 414 (2002).

28. H. J. Wang, W. H. Zhou, X. F. Yin, Z. X. Zhuang, H. H. Yang, and X. R. Wang, J. Am. Chem. Soc., 128, 15954 (2006). 